\title{
Diagnóstico que permita detectar fortalezas y debilidades para ofrecer la modalidad mixta en el Instituto Tecnológico de Altamira
}

\section{Diagnosis to detect strengths and weaknesses to offer the mixed mode at the Instituto Tecnológico de Altamira}

CASTILLO-FLORES, Ángela Liliana†, ARELLANO-BRIONES, Olimpia Danáe, ÁNGELESRESÉNDIZ, Luis Alfredo y FERNÁNDEZ-GARCÍA, Luis Guillermo

\begin{abstract}
Tecnológico Nacional de México. Av. Universidad 1200, Col. Xoco, Ciudad de México, Alcaldía Benito Juárez C.P. 03330. Instituto Tecnológico de Altamira. Carr. Tampico - Mante km 24.5, Sin Colonia, 89600 Altamira, Tamps. México.

Universidad Tecnológica de Altamira. Blvd. de los Ríos Km. 3+100, Puerto Industrial, 89603 Altamira, Tamps. México.
\end{abstract}

ID $1^{\text {er }}$ Autor: Ángela Liliana, Castillo-Flores / ORC ID: 0000-0002-8987-4526, arXiv ID Author: acastillo, CVU CONACYT ID: 298031

ID $1^{\text {er }}$ Coautor: Olimpia Danáe, Arellano-Briones / ORC ID: 0000-0001-7418-4664, CVU CONACYT ID: 246923

ID $2^{\text {do }}$ Coautor: Luis Alfredo, Ángeles-Reséndiz, / ORC ID: 0000-0002-3972-2022, arXiv ID Author: langeles, CVU CONACYT ID: 951392

ID $3^{\text {er }}$ Coautor: Luis Guillermo, Fernández-García / ORC ID: 0000-0002-6174-7656, arXiv ID Author: Luis.fernandez, CVU CONACYT ID: 879428

DOI: $10.35429 / J U P .2019 .8 .3 .7 .18$

Recibido: 11 de Abril, 2019; Aceptado 25 de Junio, 2019

\section{Resumen}

Actualmente nuestro país tiene una cobertura de $38.4 \%$, quedando diez puntos por debajo de la media de América Latina y el Caribe(UNESCO, 2018), eso significa que hasta ahora solo se ha atendido poco más de la tercera parte de la demanda social o población objetivo. Con respecto al estado de Tamaulipas, en el año 2017 se encontraba por debajo de la media, ampliando en 15.5 puntos porcentuales su brecha de cobertura (ANUIES, 2018). Una manera de aumentar la cobertura es haciendo uso de procesos educativos innovadores (Mortis Lozoya, Del Hierro Parra, García López, \& Manig Valenzuela, 2015). Los objetivos de este trabajo son: Realizar un diagnóstico que permita detectar fortalezas y debilidades para ofrecer la modalidad mixta en el Instituto Tecnológico de Altamira. Para ello se realizará una encuesta a los actores involucrados en este proceso, los estudiantes, los docentes, y las autoridades.

Modalidad Mixta, Competencias Docentes, ITA

\begin{abstract}
Currently our country has a coverage of $38.4 \%$, leaving ten points below the average for Latin America and the Caribbean (UNESCO, 2018), that means that so far only a little more than a third of the social demand has been met or target population. With respect to the state of Tamaulipas, in 2017 it was below average, widening its coverage gap by 15.5 percentage points (ANUIES, 2018). One way to increase coverage is to use innovative educational processes (Mortis Lozoya, Del Hierro Parra, García López, \& Manig Valenzuela, 2015). The objectives of this work are: Make a diagnosis to detect strengths and weaknesses to offer the mixed modality at the Technological Institute of Altamira. For this, a survey will be carried out on the actors involved in this process, the students, the teachers, and the authorities.
\end{abstract}

Citación: CASTILLO-FLORES, Ángela Liliana, ARELLANO-BRIONES, Olimpia Danáe, ÁNGELES-RESÉNDIZ, Luis Alfredo y FERNÁNDEZ-GARCÍA, Luis Guillermo. Diagnóstico que permita detectar fortalezas y debilidades para ofrecer la modalidad mixta en el Instituto Tecnológico de Altamira. Revista de Políticas Universitarias. 2019. 3-8: 7-18.

\footnotetext{
$\dagger$ Investigador contribuyendo como primer autor.
} 


\section{Introducción}

La cobertura se entiende como la magnitud en que se atiende a la población que debería estar cursando los estudios pertinentes (Fassnacht, 2017) y esta cobertura educativa se encuentra asociada al nivel de desarrollo de las entidades federativas (Fassnacht, 2017; Gil, 2009).

El gobierno, a través del plan de desarrollo Nacional en México 2019-2024, contempla en sus objetivos, el garantizar el derecho que tienen los jóvenes al acceso a la educación, destacando en éste, la ampliación de la cobertura en la educación superior. Actualmente nuestro país tiene una cobertura de $38.4 \%$, quedando diez puntos por debajo de la media de América Latina y el Caribe (UNESCO, 2018), eso significa que hasta ahora solo se ha atendido poco más de la tercera parte de la demanda social o población objetivo. El estado de Tamaulipas, en el año 2000, era el estado con la segunda mayor tasa de cobertura nacional, sin embargo en el año 2017 se encontraba por debajo de la media, ampliando en 15.5 puntos porcentuales su brecha de cobertura (ANUIES, 2018).

Una manera de aumentar la cobertura es haciendo uso de procesos educativos innovadores sin que estos demeriten la calidad del aprendizaje (Mortis Lozoya, Del Hierro Parra, García López, \& Manig Valenzuela, 2015). Rosales-Gracia, et.al. (2008) señalan que actualmente hay una amplia gama de combinaciones de los métodos de enseñanaza y resume las modalidades de los cursos en presencial o tradicional, haciendo uso de la World Wide Web (www); mixta (híbrida, blearning) presencial/en línea, y completamente en línea (asíncrona).

Integrantes de la academia de Ingeniería Industrial del plantel, Instituto Tecnológico de Altamira, pretenden integrar una propuesta de trabajo para ofertar el plan de Estudios de Ingeniería Industrial en la modalidad a distancia (entendida ésta como no escolarizada a distancia y mixta), el presente trabajo pretende dar respuesta a una pregunta decisiva ¿cuáles son las fortalezas y debilidades encontradas en el I.T. de Altamira, que se involucran en el proceso de enseñanza - aprendizaje para que el centro escolar esté en condiciones de ofertar el plan de estudios de la carrera de Ingeniería Industrial en la modalidad Mixta?
Los objetivos de este trabajo son:

Realizar un diagnóstico que permita detectar fortalezas y debilidades para ofrecer la modalidad mixta en el Instituto Tecnológico de Altamira. Para ello se realizará una encuesta a los actores involucrados en este proceso, los estudiantes, los docentes, y las autoridades.

\section{Marco Teórico}

La educación a distancia es una modalidad en educación en donde permite la inclusión de más personas que por alguna cuestión les imposibilita o limita asistir al campo de estudio, por cuestiones de tiempo, de lugar o por cuestiones laborales (Contreras, González, y Fuentes 2011). En ella, se utiliza, se emplea y se explota todo el potencial de las Tecnologías de Información y Comunicación (TIC's) en los ambientes educativos (Rosales-Gracia, GómezLópez, Durán-Rodríguez, Salinas-Fregoso, \& Saldaña-Cedillo (2008). Entre los beneficios que se observan de ofertar a los alumnos la educación a distancia es que ésta permite hacer frente a las limitaciones que se encuentran en la enseñanza tradicional, como los límites de espacio y tiempo (Contreras, González, y Fuentes 2011; Mariño,2006).

En el Decreto que crea el Tecnológico Nacional de México (TecNM) en su artículo $2^{\circ}$. Fracciones V y VIII señala que el TecNM tiene por objeto ofrecer la más amplia cobertura educativa y para ello tiene planes y programas de estudio en la modalidad denominada educación a distancia, entre ellos se encuentra la modalidad no escolarizada a distancia y mixta, conocida también como modalidad B-Learning, híbrida.

\section{¿Qués es la modalidad mixta?}

De manera simple se puede definir que el proceso de enseñanza aprendizaje impartido en la modalidad mixta, combina una modalidad de enseñanza y aprendizaje presencial tradicional con una modalidad de enseñanza y aprendizaje virtual o en línea, basado o fundamentado con el uso de medios tecnológicos (Vera, 2008). 
El Modelo de Educación Superior a Distancia del Tecnologico Nacional de México, en su versión ejecutiva (2015) define la formación profesional no escolarizada a distancia y mixta de la siguiente manera: La Formación profesional en la modalidad no escolarizada a distancia, se refiere a la formación y desarrollo de competencias profesionales del estudiante del TecNM con el apoyo del asesor(a) - tutor(a), sin necesidad de asistir a la Institución, apoyados con las Tecnologías de información y comunicación, (tutoría virtual, asesoría en línea, prácticas virtuales, plataforma educativa, etc). La formación profesional en la modalidad mixta, representa la formación y desarrollo de competencias profesionales del estudiante del TecNM en la que se requiere que reciba conducción de un docente asesor(a) tutor(a) en la institución, pero el número de horas sea menor al establecido en la modalidad escolarizada. Se puede entender que la modalidad a distancia consiste en un proceso educativo no escolarizado que incluye actividades académicas cien por ciento virtuales y/o on line, por otro lado, la modalidad mixta (híbrida) es la combinación de actividades virtuales (on-line) y manera presencial presencial, el cual está orientada a los estudiantes que adquieren una formación sin necesidad de asistir al campo institucional.

Un requisito para trabajar bajo la modalidad a distancia en la educación, es contar con una plataforma que facilite la comunicación en línea en ese prceso. Contreras, González, y Fuentes (2011) destacan que las plataformas gestoras de aprendizaje "facilitan mucho la incorporación de herramientas y recursos en línea, como apoyo al aprendizaje en las aulas. Por tanto, se hace, técnicamente, muy viable la implementación del modelo b-learning (mixto) como innovación de la práctica educativa en educación superior." Diversos autores señalan que una alternativa adecuada, viable y efectiva es utilizar plataformas o software libre, disponible gratuitamente en internet (Mariño, 2006; Esperón-Hernández \& Fuentes 2005) entre las que se pueden mencionar: Schoology, Jimdo, Claroline, Dropbox, Moodle,etc.
El lineamiento $\mathrm{N}^{\circ} 16$, Operación de la Educación a Distancia, del Manual de Lineamientos Académicos de los Institutos Tecnológicos señala que la modalidad mixta debe cubrir un número de horas mínimo bajo la conducción de un académico (presencial) y éste debe ser menor al establecido que la modalidad presencial, facilitando así el logro del aprendizaje esperado. Tanto para la modalidad no escolarizada a distancia, como para la mixta la carga académica mínima es de doce créditos y la carga máxima es de treinta y seis créditos ${ }^{1}$, y el tiempo máximo para concluir los estudios de nivel licenciatura es de 8 años o su equivalente a 16 semestres. $^{2}$ (2400 horas, para nivel Licenciatura.).

En la modalidad abierta, la interacción entre el estudiante y el Instituto, se basa fundamentalmente en documentos impresos y en asesoría presencial; en la modalidad virtual, se basa en el uso de medios informáticos y en asesoría a través de Internet o intranet. Sin embargo, ambas modalidades se pueden combinar entre sí para formar modelos mixtos.

A pesar de que el manual de lineamientos académicos contempla solamente a la educación a distancia como modalidad no escolarizada a distancia, y la mixta, el Modelo de Educación Superior a distancia del Tecnológico Nacional de México, define al modelo mixto como una combinación de la modalidad abierta y la modalidad virtual. Por ende para este trabajo denominaremos la modalidad mixta estará contemplando el modelo no escolarizado haciendo uso de asesorías presenciales mínimas al escolarizado, asesorías a distancia y haciendo uso de las Tecnologías de comunicación e información (TIC's) necesarias para alcanzar los aprendizajes y las competencias esperadas.

Sin embargo a pesar de que estas modalidades están contempladas en los lineamientos del TecNM, y como ya se mencionó anteriormente, está dirigido a personas que por razones laborales, geográficas o de marginación, no pueden asistir regularmente a clases, se observa un porcentaje relativamente bajo de programas de estudio en los institutos que se ofrecen este tipo de modalidad, predominando así el modelo tradicional, el modelo presencial.

\footnotetext{
${ }^{1}$ Manual de lineamientos académico TecNM numeral 16.4.1.8
}

${ }^{2}$ Manual de lineamientos académico TecNM numeral 16.4.1.7

CASTILLO-FLORES, Ángela Liliana, ARELLANO-BRIONES, Olimpia Danáe, ÁNGELES-RESÉNDIZ, Luis Alfredo y FERNÁNDEZ-GARCİA, Luis Guillermo. Diagnóstico que permita detectar fortalezas y debilidades para ofrecer la modalidad mixta en el Instituto Tecnológico de Altamira. Revista de Políticas Universitarias. 2019 
De los 254 institutos Tecnológicos que hay en el país, solo 44 de ellos ofrecen la modalidad de educación a distancia ${ }^{3}$, comprendidos entre aproximadamente 150 programas de estudio, un porcentaje menor al 20 por ciento.

\section{Evaluación de la educación a distancia}

Entre los indicadores coincidentes para evaluar la Educación a Distancia en México por diversos organismos institucionales se encuentran; Las Políticas, objetivos y estrategias institucionales; La Identificación de las principales fortalezas y problemas del programa en el ámbito institucional en las modalidades a distancia mediadas por TIC; los estudiantes; el personal académico, los servicios de apoyo (a estudiantes y docentes), la Infraestructura y los servicios, entre otros indicadores (México, 2015)

Para poder impulsar el uso de está modalidad es menester que las autoridades pertinentes de cada instituto en conjunto con las academias, establezcan un diagnóstico y un plan rector estratégico y táctico dentro de la institución (Pacheco \& Robles, 2006).

Por ello este documento pretende realizar un diagnóstico, el cual se basa en 3 de las dimensiones más sobresalientes, comunes para la evaluación de la educación en la modalidad analizada, y que son considerados entre otros, como marco de referencia esenciales para garantizar y evaluar la calidad de la educación a distancia y mixta, estas dimensiones son las: las Políticas, objetivos y estrategias institucionales; la Infraestructura y el equipamiento; los docentes y los estudiantes (Rocha, Maina, \& Sangra, 2013; Farfán, 2016)

\section{Las políticas y estrategias institucionales}

Aunque están creados los lineamientos de la educación a distancia en los institutos tecnológicos, Francisco (2012) señala que para alcanzar el éxito en las modalidades a distancia y virtuales la voluntad política es imprescindible, manifiesta que las autoridades de las instituciones deben ser los primeros actores en estar comprometidos con el éxito de la modalidad ya que de ellos depende promover la gestión de los recursos técnicos y sociales necesarios (infraestructura, equipamiento, capacitación docente, etc) y también deben motivar la incorporación activa y consciente de todos los miembros de la comunidad educativa en alcanzar los objetivos del programa bajo esta modalidad para poder cumplir con estándares de calidad requeridos. Por su parte López González \& Ramírez Martinell (2016) señala que la institución debe crear lineamientos claros para todos los procesos, incluyendo el proceso de esta modalidad mixta, atribuye incluso que la falta de interés por parte de la administración crea un desconocimiento y una falta de interés por parte de los profesores sobre el uso educativo eficiente de la tecnología.

Para introducir está modalidad en las instituciones Pacheco \& Robles, (2006) recomiendan que se debe integrar un equiporector multidisciplinario, iniciando con la voluntad de la administración, y manifestándose mediante un plan rector estratégico y táctico dentro de la institución.

Con el objetivo de conocer los objetivos a corto y mediano plazo al interior del instituto, se realizó una entrevista con las autoridades correspondientes, así también para indagar la voluntad que se tiene para introducir está modalidad mixta dentro de la institución, y para establecer, desde su perspectiva, las fortalezas y debilidades del Instituto ante está necesidad de ofertar la modalidad mixta en el programa de Ingeniería Industrial.

\section{Infraestructura y equipamiento}

En este apartado se señala la necesidad de la infraestructura física $\mathrm{y}$ el equipamiento tecnológico requerido para transitar por la modalidad mixta.

\footnotetext{
3 Tomado de: https://www.tecnm.mx/docencia/reunionnacional-de-educacion-a-distancia 
Según el manual de lineamientos de la educación a distancia del TecNM la Infraestructura considera los siguientes elementos: "Las unidades de educación a distancia regionales y locales, las cuales son instalaciones en las que se proporcionan materiales, equipo y conectividad para que los estudiantes fortalezcan las competencias profesionales. Otros elementos, como la Institución y los recursos tecnológicos, son instalaciones físicas o de comunicaciones que dan soporte y auspician al proceso de enseñanza-aprendizaje". Sin embargo, para Bates (2001) el término Infraestructura abarca no sólo el aspecto físico sino también el humano, el financiero, el tecnológico, de accesibilidad, entre otros aspectos. En esta investigación hace referencia a la Infraestructura física vinculando los elementos de accesibilidad, conectividad, espacios. En lo referente a equipamiento, este término abarca equipos de cómputo (con cámaras, micrófono), acervos digitales y licencias de uso de software, software especializado (CAD-simulación, etc); proyectores, conexión a internet (alámbrica e inalámbricamente), servidor y hospedaje, administración e instalación de plataforma educativa y entorno virtual (López González\& Ramírez Martinell, 2016).

Por tanto, en la encuesta diagnóstico, desde la perspectiva de los actores involucrados: autoridades, docentes y estudiantes, se indagará si ellos consideran si la institución cuenta con la infraestructura y el equipamiento mínimo necesario que pueda dar soporte al trabajo educativo en línea.

El Personal académico, el Docente.

Aunque el Modelo de Educación a distancia del TecNM, en su apartado 1.4.4, y 1.4.5 define las políticas de operación y/o funciones atribuidas al docente-asesor y docente- tutor, en la modalidad en línea, y de allí se extraen las características y atribuciones del docente, las cuales contempla: el manejo de las TIC's, la elaboración de material didáctico, actividades e instrumentos de evaluación, entre otras actividades. Sin embargo, este mismo lineamiento atribuye que la adaptación de está modalidad en los institutos deberá ser una característica que se desarrollará desde la implementación en cada uno de ellos, a través de la participación de los docentes, directivos y estudiantes.
Por tanto, para la realización del diagnóstico, los autores de este trabajo consideran las competencias sugeridas por Céspedes, Matarrita, \& Castro (2010), ya que se observó que éstas abarcan los atributos marcados en los lineamientos del TecNM, y a su vez considera otras habilidades necesarias para lograr una mayor efectividad en el proceso educativo a distancia. Por tanto, a partir de ésta propuesta se analizan las fortalezas y debilidades de los docentes dentro del Instituto Tecnológico de Altamira, abarcando las competencias en cinco ámbitos: el tecnológico, el pedagógico, el social, el investigativo y de liderazgo. En la Figura 1, describe el perfil de las competencias del docente en la modalidad en línea sugeridas por Céspedes, et.al (2010).

A continuación, se define brevemente cada una de ellas.

1. Competencias Tecnológicas: Referidas a las habilidades para la aplicación de herramientas tecnológicas asociadas con el proceso educativo.

2. Competencias Investigativa: Comprende el conjunto de habilidades, conocimientos y actitudes que le permiten la problematización de la realidad educativa y la búsqueda sistemática de respuestas.

3. Competencias Pedagógicas: Implica el dominio de teorías de enseñanza y aprendizaje y de diseño instruccional, que permiten el planteamiento, desarrollo $\mathrm{y}$ evaluación de propuestas pedagógicas efectivas. 


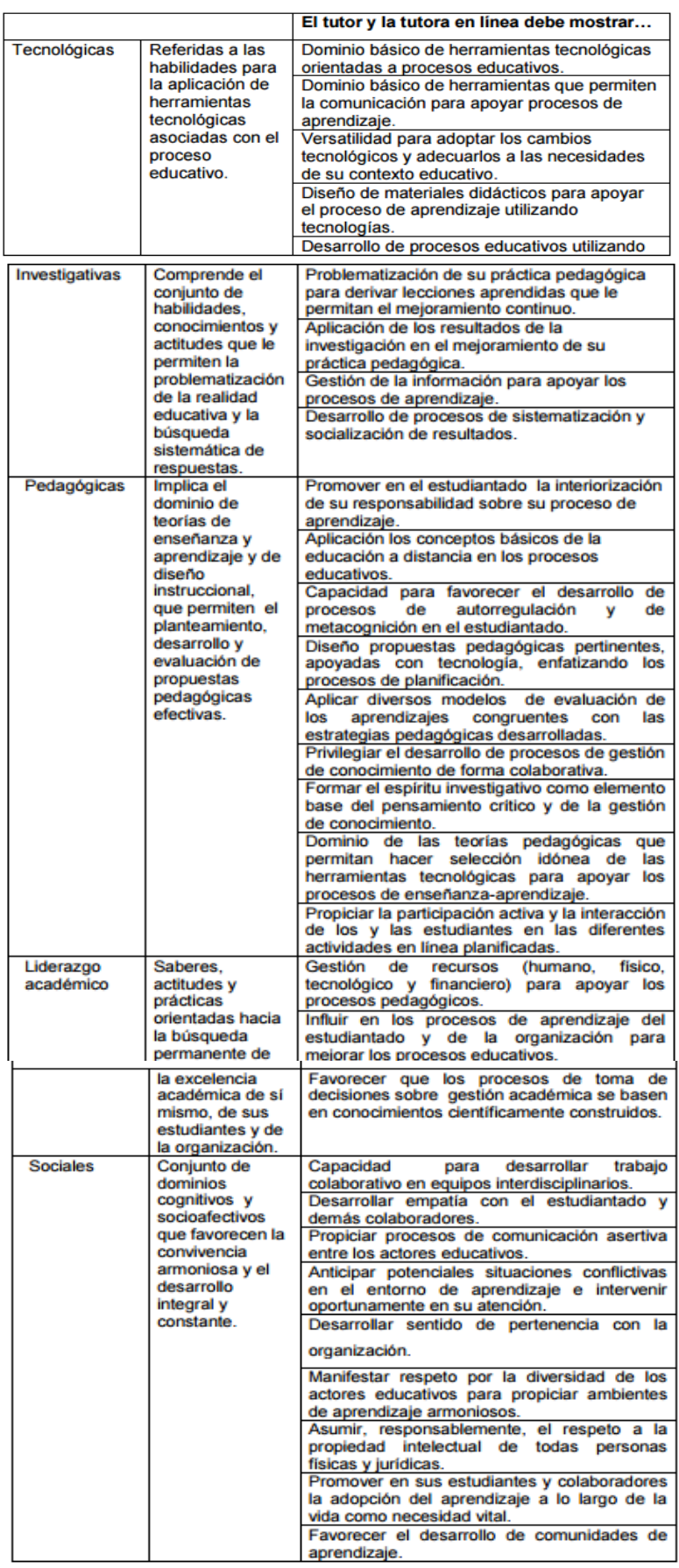

Tabla 1 Perfil de competencias para el docente de educación superior

Fuente: Céspedes, Matarrita, \& Castro (2010)

4. Competencia de liderazgo académico: Saberes, actitudes y prácticas orientadas hacia la búsqueda permanente de la excelencia académica de sí mismo, de sus estudiantes y de la organización.

5. Competencia social: Conjunto de dominios cognitivos y social-afectivos que favorecen la convivencia armoniosa y el desarrollo integral y constante.

\section{Método}

Diseño transversal descriptivo. Aplicación de una encuesta dirigida a los estudiantes y profesores de la carrera de Ingeniería Industrial.

Población:

Estudiantes Inscritos de la Carrera de Ingeniería Industrial, 401, alumnos

Docentes que imparten cátedra en el programa de Ingeniería Industrial

Muestra:

Muestra alumnos $30 \%$.

Muestra de docentes: $20 \%$

Instrumento:

Basado en la teoría antes mencionada, se diseño un cuestionario dirigido a los docentes, estudiantes y una entrevista dirigida a las autoridades de la institución.

\section{Resultados}

En este apartado se muestra los resultados de los aspectos más destacados de las encuestas realizadas.

\section{Estudiante}

El 65\% de los encuestados son hombres y el $35 \%$ son mujeres (ver gráfico 1).

\section{Sexo}

87 respuestas

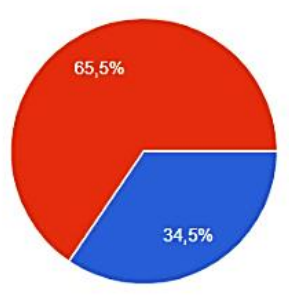

- Mujer

Hombre

Gráfico 1 Porcentaje de Hombres y mujeres

Fuente: elaboración propia

Se observó que casi el $40 \%$ de los estudiantes trabajan (ver Gráfico 2). 
Actualmente ¿trabajas?

87 respuestas

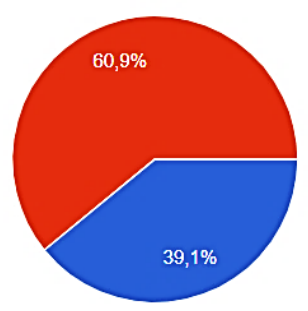

Ni

Gráfico 2 Estudiantes trabajando

Fuente: elaboración propia

Aproximadamente el $40 \%$ de los estudiantes encuestados trabajan y el $60 \%$ estudian solamente.

Con respecto a conocer si los estudiantes tienen familia, los resultados arrojaron que el 95\% de los encuestados no tiene hijos y tan solo el 5\% si los tiene (ver Gráfico 3).

\section{¿Tienes hijos?}

87 respuestas

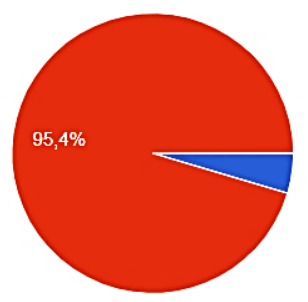

Si

Gráfico 3 Estudiantes con hijos

Fuente: elaboración propia

En cuestión de costo de transporte los estudiantes respondieron que: aproximadamente el $44 \%$ menciona destinar del 25 al $50 \%$ de sus gastos al transporte. (ver Gráfico 4)

¿Qué porcentaje de tu gasto se destina al transporte? 87 respuestas

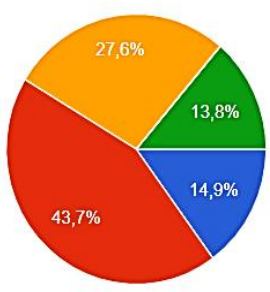

- 0 a $25 \%$ 50 a $75 \%$ - 75 a $100 \%$
Gráfico 4 Gasto en transporte Fuente: elaboración propia
En cuestión de conocer si los estudiantes encuestados cuentan con computadora y acceso a internet, los datos arrojaron lo siguiente:

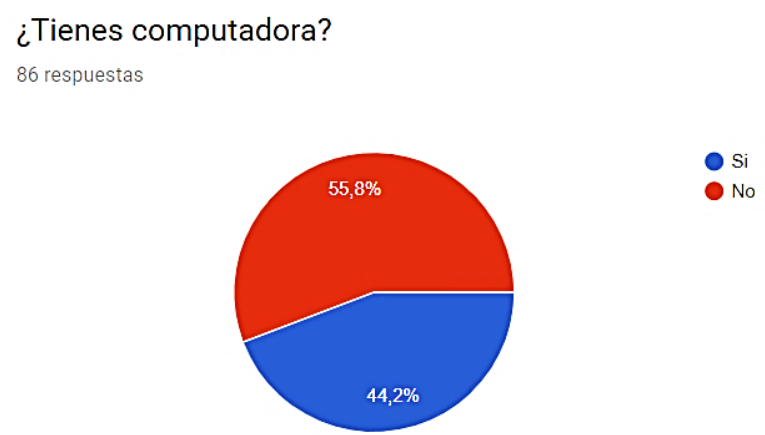

Gráfico 5 Estudiantes con equipo de computo Fuente: elaboración propia

El $44 \%$ de los encuestados cuentan con computadora. (ver Gráfico ).

¿Tienes acceso a internet? 87 respuestas

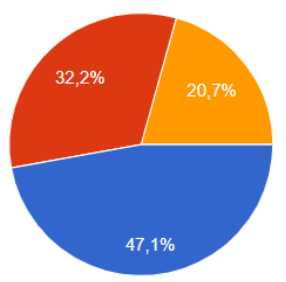

Gráfico 6 Estudiantes con acceso a internet Fuente: elaboración propia

El $47 \%$ cuenta con acceso a internet, el $32 \%$ no tiene acceso y el $21 \%$ menciona tener a veces acceso a internet (ver Gráfico 6). Al preguntar de manera directa a los estudiantes si ellos consideran que es pertinente combinar la educación a distancia y en línea se observó que:

Por tus circunstancias de familia, trabajo o distancia, ¿consideras la pertinencia de combinar la educación en línea y presencial (asistir menos días a la semana)?

87 respuestas
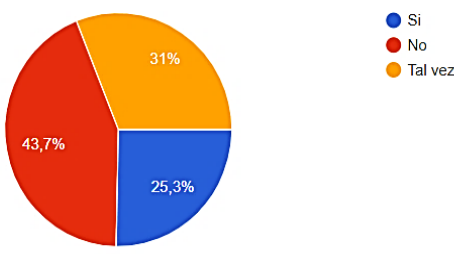

Gráfico 7 Pertinencia de la educación a distancia

El $25 \%$ de los encuestados considera pertinente una modalidad en línea y el $31 \%$ lo consideran como tal vez (ver Gráfico 7). 


\section{De los Docente}

A continuación se muestran parte de los resultados obtenidos desde la perspectiva del docente, en la encuesta aplicada se consideraron las características y/o competencias propuestas por Céspedes, Matarrita, \& Castro (2010), abarcando cinco ámbitos: el tecnológico, el pedagógico, el social, el investigativo y de liderazgo, agregando una sección para conocer su perspectiva y disposición que tiene el docente para introducir la modalidad mixta en su centro de trabajo.

\section{Investigativa.}

En la característica investigativa, se observó que la mayoría de las personas, casi 35 $\%$ respondieron la encuesta han realizado sólo una publicación, y un 25 por ciento mencionó no haber realizado publicaciones (ver Gráfico 8).

Si realiza usted investigación ¿Cuántas publicaciones ha realizado en los últimos tres años? 12 respuestas
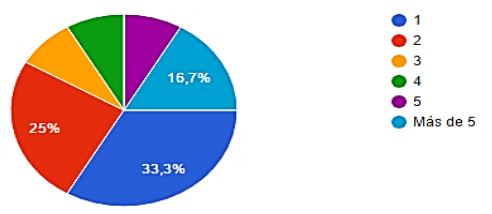

¿Ha asistido a congresos como ponente?

Gráfico 8 Docentes realizando investigación

Fuente: elaboración propia

Por otro lado, se observa que la mayoría de las personas que respondieron la encuesta han asistido a un congreso como participante (ver Gráfico 9).

¿Ha asistido a congresos como ponente? 15 respuestas

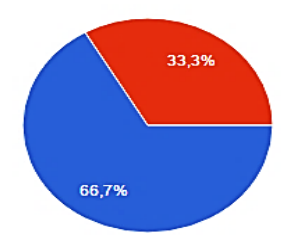

in si

¿A cuantos congresos ha participado en los últimos 3 años? 14 respuestas

Gráfico 9 Docentes que han asistido a congresos

Fuente: elaboración propia
Pedagogía.

En el ámbito de la pedagogía se puede observar que la mayoría de los docentes no tienen algún curso pedagogico, aproximadamente un 70\% (ver Gráfico 10).

¿Tiene usted cursos de pedagogía?

15 respuestas

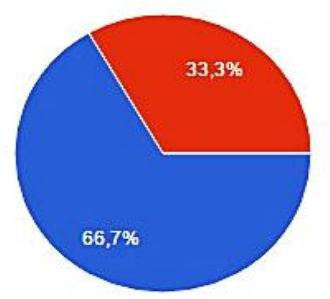

Gráfico 10 Docentes con cursos de Pedagogía

Fuente: elaboración propia

Liderazgo y Social.

En la cuestión social se observa que la mayoría de los docentes no ha lidereado ningún proyecto académico (ver Gráfico 11).

¿Ha sido líder en algún proyecto académico?

15 respuestas

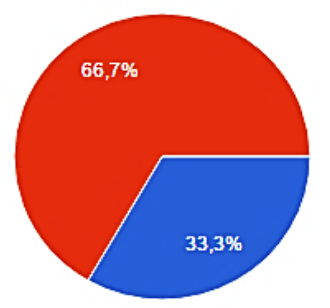

Gráfico 11 Liderazgo

Fuente: elaboración propia

En la cuestión social se puede decir que el $100 \%$ de los docentes pertenecen alguna academia (ver Gráfico 12).

En la institución ¿Pertenece por lo menos a una academia de maestros? 15 respuestas

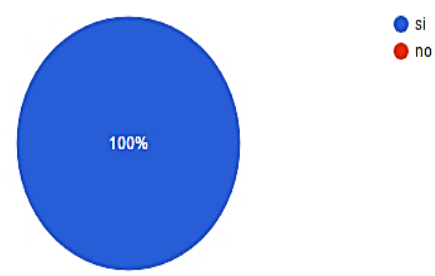

Gráfico 12 Docentes que pertenecen a academia Fuente: elaboración propia 


\section{Tecnología.}

En la cuestión tecnológica se puede decir que más del $90 \%$ que respondieron la encuesta han desarrollado material didáctico en su proceso de enseñanza aprendizaje (ver Gráfico 13).

¿Ha desarrollado materiales didácticos para apoyar el proceso de aprendizaje utilizando tecnologías de la Información y/o comunicación (manera digital, multimedi en formatos, Word, Power, Flash, HTML, etc.)?

15 respuestas

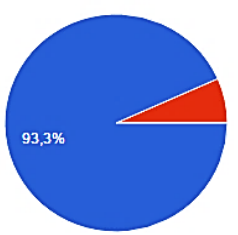

\section{si}

Gráfico 13 Desarrollo de material didáctico

Fuente: elaboración propia

Con respecto a la utilización de las TIC's se puede decir que todos los docentes las han utilizado en su proceso de enseñanza aprendizaje (ver Gráfico 14).

¿Utiliza herramientas de las TIC's en su práctica docente?

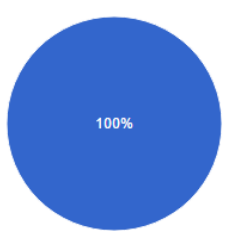

- si

Gráfico 14 Utilización de TIC's

Fuente: elaboración propia

Se observa que alrededor del $40 \%$ han impartido cursos en línea (ver Gráfico 15).

¿Ha impartido cursos en línea?

15 respuestas

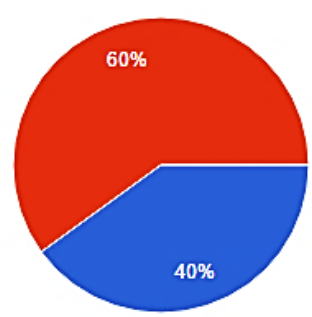

Gráfico 15 Impartición de cursos en línea Fuente: elaboración propia
Sin embargo, más del $90 \%$ si han participado como estudiantes en cursos en línea (ver Gráfico 16).

¿Ha participado como estudiante en cursos en línea? 15 respuestas

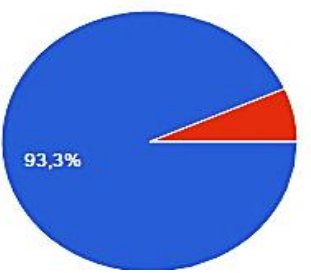

Gráfico 16 Estudiante cursos en línea

Fuente: elaboración propia

Perspectiva.

Se observa que la un $80 \%$ de los docentes si les interesa impartir cursos en la modalidad mixta (ver Gráfico 17).

¿Le interesaría impartir cursos y/o materias a distancia en su modalidad mixta?

15 respuestas
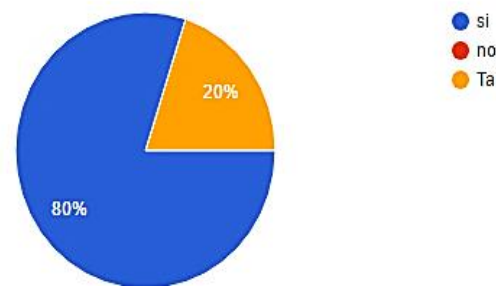

Tal vez

Gráfico 17 Interés del docente por impartir cursos en línea Fuente: elaboración propia

De los docentes que están dispuestos en impartir cursos de la modalidad mixta, más del $90 \%$ de ellos están dispuestos a adoptar cambios tecnológicos y adecuarlos a sus prácticas docentes (ver Gráfico 18).

Si está usted interesado en impartir cursos y/o materias del programa de Ingeniería Industrial en modalidad mixta ¿Está dispuesto a adoptar cambios tecnológicos y adecuarlos a las necesidades de su práctica docente?
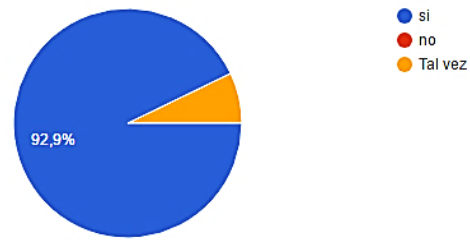

Gráfico 18 Docentes dispuestos a adoptar cambios tecnológicos en su práctica docente

Fuente: elaboración propia 
Con respecto al interés en colaborar en el diseño de los materiales didácticos requeridos para impartir la modalidad mixta, se observa que el $100 \%$ de las personas encuestadas si están dispuestos a colaborar con esa tarea (ver Gráfico 19).

Si está usted interesado en impartir cursos y/o materias del programa de Ingeniería Industrial en la modalidad mixta ¿Está dispuesto a colaborar en el diseño de los materiales didácticos requeridos para impartir las materias en esta modalidad mixta?
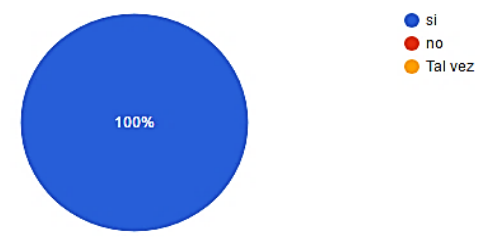

Gráfico 19 Diseño de materiales didácticos Fuente: elaboración propia

En matera de recibir capacitación, se observó que casi un $90 \%$ de los docentes encuestados participarían en las capacitaciones requeridas para impartir está modalidad. (ver Gráfico 20)

Si está usted interesado en impartir cursos y/o materias del programa de Ingeniería Industrial en la modalidad mixta ¿Participaría usted en las capacitaciones requeridas para los docentes en está modalidad? 15 respuestas

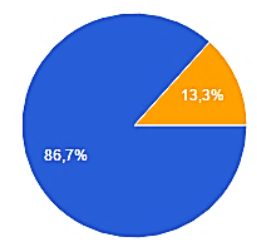

\section{no}

Tal vez

Gráfico 20 Disposición del docente a capacitarse Fuente: elaboración propia

Se observa que la mayoría de los docentes consideran que la conectividad no es la adecuada para impartir la modalidad a mixta (ver Gráfico 21).

Consideras que la conectividad a internet en la institución es la adecuada para tomar y/o impartir cursos en línea.

15 respuestas

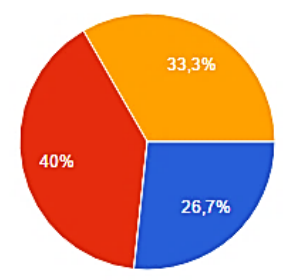

si

Tal vez

Gráfico 21 Percepción del docente respecto a la conectividad de la institución

Fuente: elaboración propia
Con respecto a la Infraestructura, más del $70 \%$ considera que el instituto tiene la infraestructura mínima requerida para ofertar la modalidad mixta en el instituto (ver Gráfico 22).

¿Consideras que se cuenta con la infraestructura mínima requerida para ofertar el programa de Ingeniería Industrial en la modalidad mixta? 15 respuestas

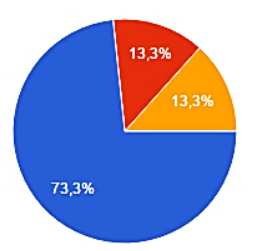

- si

Tal vez

Gráfico 22 Percepción del docente respecto a la Infraestructura de la institución

Fuente: elaboración propia

\section{De la Administración}

Derivado de la entrevista a las Autoridades Institucionales: MC. Miguel Ángel Villar Morales, Director del Instituto Tecnológico de Altamira, y de la MC. Gabriela Del Pilar Gómez Mendoza, Subdirectora Académica, sus reflexiones van encaminadas a los ejes transversales de la Administración Federal: cobertura e inclusión, además señalan que, el crecimiento natural de la matrícula del Programa del estudio (Ingeniería Industrial), hace necesario la diversificación de los servicios educativos a fin de satisfacer la demanda en el acceso a la Educación Superior Pública, convirtiéndose esto en uno de los objetivos institucionales en el corto y mediano plazo. Aunado a lo antes descrito, estas autoridades reconocen que entre los retos institucionales para la apertura de esta modalidad son de Infraestructura Tecnológica, Personal Docente Capacitado en el modelo y la elaboración del Diseño Instruccional por parte de la academia, entre otros rubros. Sin embargo, manifestaron la voluntad y el compromiso que tiene está administración en apoyar el desarrollo de la apertura no solo del programa de Ingeniería Industrial, sino también de las demás carreras previa análisis de pertinencia en cada una de ellas.

\section{Conclusiones}

De los estudiantes, De acuerdo con los datos obtenidos, se puede considerar pertinente la apertura de la modalidad mixta, ya que un porcentaje significativo de alumnos cuenta con las condiciones mínimas requeridas para poder cursar tal modalidad.

CASTILLO-FLORES, Ángela Liliana, ARELLANO-BRIONES, Olimpia Danáe, ÁNGELES-RESÉNDIZ, Luis Alfredo y FERNÁNDEZ-GARCİA, Luis Guillermo. Diagnóstico que permita detectar fortalezas y debilidades para ofrecer la modalidad mixta en el Instituto Tecnológico de Altamira. Revista de Políticas Universitarias. 2019 
Por otro lado, un porcentaje significativo de estudiantes considera factible y que además están interesados en la apertura de la modalidad mixta. De los Docentes, en base a los resultados obtenidos en la encuesta aplicada a los docentes se puede concluir que existe disposición por parte de ellos en colaborar activamente en este proyecto, ya que la mayoría de las personas que respondieron la encuesta no solo están interesados en impartir cátedra en esa modalidad sino que están dispuestos en elaborar material didáctico requerido para la apertura de la modalidad mixta y también, están dispuesto a recibir capacitación necesaria para ello.

Al establecerse, la necesidad de esta modalidad por parte del estudiante, al determinar que casi $40 \%$ de ellos trabajan y que la mayoría de éstos mencionaron gastar mucho recurso en pasajes, por la distancia que tienen que recorrer para llegar al instituto, también se descubrió que casi una tercera parte de ellos les gustaría cursar en la modalidad mixta; en cuestión de los docentes se pudo observar la disposición y el interés que ellos tienen en participar en esta modalidad, y por último se destaca por parte de las autoridades correspondientes del instituto, la voluntad y el compromiso que se tiene para respaldar la apertura de esta modalidad mixta en la institución.

\section{Recomendaciones}

Según los resultados obtenido a través de las encuestas realizadas a los estudiantes y los docentes, y la entrevista realizada a las autoridades institucionales, los autores de este trabajo concluyen que existen las condiciones básicas necesarias para la apertura de la modalidad en el programa de Ingeniería Industrial en el Instituto Tecnológico de Altamira, encontrándose a través del diagnóstico, que existe oportunidad de mejora en cuestión de la infraestructura considerando que es, la conectividad el principal elemento que se requiere prestar principal atención, sin embargo en la entrevista realizada a las autoridades, ellos destacaron que ya se está trabajando al respecto de este tema en la institución. En cuestión de las competencias requeridas por el docente en está modalidad, se observó hace falta reforzar en cursos pedagógicos, en realizar investigación y dirigir algún proyecto académico.
Por parte de la academia de Ingeniería Industrial, es menester trabajar en el material didáctico, los diseños instruccionales necesarios para arrancar el programa bajo esta modalidad, en el siguiente periodo escolar. Al analizar el manual de lineamientos de la educación a distancia del TecNM, se encontraron las siguientes En cuestión de las materias que se cursan en el programa, la academia debe establecer cuánto será la cantidad "mínimas" de horas presenciales que debe tener la (s) materia (s) en esta modalidad ya que el manual no específica ¿Cuántas son mínimas?, en este sentido los integrantes de este trabajo se apegarán a lo considerado en dicho manual, en donde se le atribuye a cada institución la adecuación de las particularidades no previstas en él. Incluso tampoco establece si todas las materias requieren asesorías presenciales no.

\section{Referencias}

ANUIES. (2018). Visión y acción 2030, Propuesta de la ANUIES para renovar la educación superior en México. Obtenido de Diseño y concertación de políticas públicas para impulsar el cambio institucional: https://visionyaccion2030.anuies.mx/Vision_ac cion2030.pdf

Bates, T. (2001), Cómo gestionar el cambio tecnológico, Barcelona, Editorial Gedisa/Ediciones UOC.

Esperón-Hernández, R. I., \& Fuentes, M. L. (2005). Educación en línea en la UADY mediante un software libre. Apertura, 67-72.

Francisco, J. (2012). Calidad en entornos virtuales de aprendizaje. Compendium, 15(29), 97-107.

Farfán, Pablo (2016): "Modelo de virtualización educativa de la universidad politécnica Salesiana del Ecuador", tesis de doctorado, Centro de Estudios para el Perfeccionamiento de la Educación Superior (CEPES), La Habana.

Rosales-Gracia, S., Gómez-López, V. M., Durán-Rodríguez, S., Salinas-Fregoso, M., \& Saldaña-Cedillo, S. (2008). Modalidad híbrida y presencial: Comparación de dos modalidades educativas. Revista de la educación superior, 37(148), 23-29. 
Contreras, L., González, K., y Fuentes, H. (2011). Uso de las TIC y especialmente del Blended Learning en la enseñanza universitaria. Revista Educación y Desarrollo Social, 5(1), 151-160. Recuperado de: http://www.umng.edu.co/documents/63968/ 76126/11.pdf [Links]

Céspedes, J. C., Matarrita, O. L. B., \& Castro, A. S. (2010). Competencias del docente de educación superior en línea. Revista Electrónica" Actualidades Investigativas en Educación", 10(3), 1-19.

Mariño, J. C. G. (2006). B-Learning utilizando software libre, una alternativa viable en Educación Superior. Revista complutense de Educación, 17(1), 121.

México, U. N. (2015). La Educación a Distancia en México: Una nueva realidad universitaria. México: Universidad Nacional Autónoma de México.

Rocha, P., Maina, M., \& Sangra, A. (2013). Análisis del Marco de referencia para la evaluación de los ambientes virtuales a nivel superior en el contexto de América Latina y Europa. Memorias del Encuentro Internacional de Educación a Distancia, (2).

Gil Antón, M (2009). Cobertura de la educación superior en México: Tendencias, retos y perspectivas, México: ANUIES.

Fassnacht, E. F. (2017). Una mirada a los desafíos de la educación superior en México. Innovación Educativa, 17(74), 183207.

Fernández-Morales, K. A. T. I. U. S. K. A., \& Vallejo-Casarín, A. L. M. A. (2014). La educación en línea: Una perspectiva basada en la experiencia de los países. Rev Educ Des, 29, 2939.

López González, R., \& Ramírez Martinell, A. (2016). Políticas de equipamiento tecnológico en Educación Superior: reflexiones y orientaciones. Debate universitario, 5(9), 53-67

Pacheco, A., \& Robles, J. (2006). M-Learning: educación y capacitación móvil.

Vera, F. (2008). La modalidad blended-learning en la educación superior. Rancagua, Chile 